\title{
¿Protegen las espinas arbustivas a los nidos de aves contra la depredación? Un análisis experimental en la estepa patagónica
}

\author{
M. Soledad Vazquez ${ }^{\bowtie}$ \& Alejandro G. Farji-Brener \\ Laboratorio Ecotono INIBIOMA, CONICET-Universidad Nacional del Comahue.
}

\begin{abstract}
Resumen. La depredación de nidos es una de las presiones de selección más importantes para las aves, por lo que la elección del sitio de nidificación es clave para la supervivencia de los polluelos. Evaluamos de manera experimental si la presencia de espinas en arbustos contribuye a la protección contra la depredación de nidos. El trabajo se desarrolló cerca de la ciudad de San Carlos de Bariloche, Argentina, en un ambiente de estepa patagónica. Colocamos nidos artificiales sobre arbustos de rosa mosqueta a los que les modificamos de forma experimental la densidad de espinas, y sobre arbustos completamente libres de espinas. La tasa general de depredación fue cercana al $90 \%$ y no resultó afectada por la densidad de espinas. Discutimos otras posibles causas de la elección de arbustos espinosos como sitios de nidificación.
\end{abstract}

[Palabras clave: estepa patagónica, microhábitat, nidos artificiales, paseriformes]

\begin{abstract}
Aвstract. Do shrub thorns protect bird nests against predation? An experimental analysis in the Patagonian steppe. Nest predation is one of the most important selection pressures for birds; so, the choice of nesting site is key to chick survival. We experimentally evaluated if the presence of thorns in shrubs contributes to the protection against nest predation. The work was developed near the city of San Carlos de Bariloche, Argentina, in a Patagonian steppe environment. We placed artificial nests on rose mosqueta shrubs, experimentally varying the density of thorns, and on shrubs naturally free of thorns. The general predation rate was around $90 \%$ and was not affected by the density of thorns. Possible causes of the selection of thorny shrubs as nesting sites are discussed.
\end{abstract}

[Keywords: artificial nests, passeriforms, microhabitat, Patagonian steppe]

\section{INTRODUCCIÓN}

La depredación de nidos fue descripta como una de las principales causas del fracaso reproductivo en diversas especies de aves. El promedio de las pérdidas que causa la depredación alcanza valores cercanos a 80\% (Lima 2009; Segura et al. 2012; Smith et al. 2016). En consecuencia, la selección del sitio de nidificación puede ser crucial para la supervivencia de su descendencia (Lima 2009; Martin and Briskie 2009). Las aves usan distintas estrategias para mejorar ese éxito reproductivo. Por ejemplo, algunas especies nidifican en las mismas ramas que nidos de avispas (Joyce 1993) o en árboles de acacia donde viven colonias de hormigas que pueden disuadir a los depredadores (Young et al. 1990). Otras ocultan sus nidos o los construyen en sitios inaccesibles para sus depredadores (Segura et al. 2012; Collias and Collias 2014). Estas estrategias muchas veces dependen no sólo del comportamiento de las especies de aves, sino también del entorno en donde estas habitan.

Editor asociado: David Bilenca 凶vazquezm@comahue-conicet.gob.ar
Durante el proceso de nidificación, las aves pueden aprovechar diversas características de las especies de plantas disponibles para reducir la probabilidad de depredación. Por ejemplo, la densidad de follaje, la altura de la rama o la presencia de espinas pueden ser condiciones que afecten la visibilidad o accesibilidad de los depredadores (Lazo and Anabalón 1991; Kelly 1993, Filliater et al. 1994; Segura et al. 2012). En particular, las espinas pueden servir de base estructural para incrementar la estabilidad de los nidos (Janzen 1969; Biddle et al. 2017) y también desalentar el acceso de ciertos depredadores (Schmidt and Whelan 1999). Sin embargo, anidar en ramas espinosas puede reducir el acceso de ciertos depredadores, pero facilitar el de otros. Por ejemplo, las espinas pueden reducir el ataque de serpientes (Quader 2006), pero resultar indiferentes, o facilitar, el acceso de aves o de roedores (Mezquida and Marone 2002; Borgmann and Rodewald 2004). Nosotros determinaremos experimentalmente la importancia de la presencia de espinas sobre la probabilidad de depredación de nidos en 
la estepa patagónica. Si las espinas reducen la probabilidad de depredación, esperamos un porcentaje menor de depredación en nidos experimentales ubicados en ramas con espinas que en ramas espinosas a las que se les removieron las espinas y en ramas que naturalmente carecen de espinas.

\section{Materiales y MÉTOdos}

\section{Área de estudio}

El trabajo fue desarrollado en la zona cercana a los ríos Limay y Nirihuau, en San Carlos de Bariloche, provincia de Río Negro, Argentina $\left(41^{\circ} 3^{\prime} 26.2^{\prime \prime} \mathrm{S}-71^{\circ} 8^{\prime} 53.5^{\prime \prime} \mathrm{O}\right)$ cuyo hábitat dominante es la estepa patagónica. El clima de la región es frío y seco, con características de semi-desierto, con precipitaciones promedio de $300 \mathrm{~mm}$ anuales. La temperatura media anual varía alrededor de $12^{\circ} \mathrm{C}$; son característicos los fuertes vientos del oeste. La estepa patagónica se compone principalmente de arbustos y matas de hierbas dispersas en una matriz de suelo desnudo (Aguiar et al. 1996). La vegetación se presenta bajo la forma de matorrales achaparrados, muchos con forma de cojín y otros espinosos (Burkart et al. 1999). La especie predominante cuando este ambiente se encuentra parcialmente disturbado por caminos, construcciones y presencia humana es la rosa mosqueta (Rosa rubiginosa, Rosaceae), un arbusto exótico cubierto de espinas que suele ser elegido por las aves del área como sitio de nidificación (ver más adelante). Las aves terrestres de la estepa suman unas 110 especies (de La Vega et al. 2003). Muchas de ellas construyen nidos en forma de taza con material vegetal y ramitas; a menudo, el interior de los nidos está tapizado con plumas o pelos (Povedano and Bisheimer 2016). Además de las aves, entre los potenciales depredadores de nidos presentes en la zona se encuentran las lagartijas, zorrinos, hurones y una gran variedad de roedores (Matteucci 2012).

\section{Diseño de muestreo}

Elaboramos 100 nidos artificiales de copa abierta simulando nidos naturales de Fio-Fio (Elaenia albiceps) y otras aves del área. Los nidos fueron construidos con un diámetro promedio de $10 \mathrm{~cm}$, una altura de $6 \mathrm{~cm}$ y con los materiales que suelen usar las aves, como fibras vegetales, pastos secos y palitos, recolectados en la zona de estudio (De la Peña 2013). Para evaluar si las espinas protegen contra la depredación de nidos ubicamos cuatro nidos en 25 cuadrantes de aproximadamente $5 \times 5 \mathrm{~m}$, distribuidos en 5 sitios en el área de estudio. Los sitios se separaron por una distancia de al menos $500 \mathrm{~m}$ y cada cuadrante dentro del sitio tenía una separación mínima de $150 \mathrm{~m}$. Cada cuadrante se caracterizó por contener un arbusto espinoso de rosa mosqueta de entre 1-3 m de altura y un arbusto sin espinas de altura y estructura similar. Si bien en la zona de estudio hay otras especies nativas con espinas, los disturbios ocasionados por el cambio en el uso de la tierra, la construcción de rutas y la urbanización facilitaron la dominancia de la rosa mosqueta. Elegimos este arbusto exótico porque en el sitio de estudio es el más abundante y representa un recurso clave para la nidificación de las aves. Los muestreos preliminares revelaron que alrededor de $80 \%$ de los nidos de aves en el sitio de estudio estaban construidos en rosa mosqueta ( $\mathrm{n}=22$ nidos) (M. S. Vazquez, datos no publicados). Las especies de aves del área que más frecuentemente emplean este arbusto para nidificar son el zorzal (Turdus falcklandii), rara (Phytotoma rara), coludito copeton (Leptasthenura platensis) y churrin grande (Eugralla paradoxa) (L. Sympson, comunicación personal). Los arbustos sin espinas utilizados fueron individuos de maitén (Maytenus boaria, Celastraceae), laura (Schinus patagonicus, Anacardiaceae), palo piche (Fabiana imbricata, Solanaceae) y vautro (Baccharis patagónica, Asteraceae). Dispusimos los cuatro nidos al azar en 4 tratamientos (un nido por tratamiento). Los 3 primeros tratamientos los realizamos en la rosa mosqueta y fueron denominados "con espinas" (E), "con espinas agregadas" (+E) y "espinas removidas" (-E). El primero (E) consistió en colocar un nido en la rosa mosqueta sin hacer ninguna modificación. Para el segundo $(+\mathrm{E})$ cortamos ramas de otro arbusto de rosa mosqueta y las añadimos al arbusto experimental de tal manera de aumentar un 100\% la densidad de espinas alrededor del nido colocado. Por último, el tratamiento -E consistió en quitar de forma manual todas las espinas en un área de 25x25 cm alrededor del nido. Para el cuarto tratamiento colocamos un nido en el arbusto sin espinas, a la misma altura y ubicación que los otros tratamientos. Ubicamos los nidos entre 0.5 y 2 m de altura y evitamos colocarlos en la periferia del arbusto. En este sentido, los nidos se ubicaron a una distancia al interior de la periferia similar en todos los casos, con lo cual se mantuvo una 'accesibilidad' semejante entre los tratamientos para los eventuales 
depredadores. Cada nido contenía un huevo de canario (Serinus canaria, Fringillidae), que posee un tamaño similar a los de las especies que nidifican en la zona y que evita subestimar pequeños depredadores mamíferos (Maier and DeGraaf 2000), y un huevo de plastilina deigual dimensión y color gris. El huevo de plastilina se colocó para intentar determinar la identidad del depredador por las marcas en la plastilina. Experimentos preliminares confirmaron que los huevos de plastilina podrían ser detectados y atacados por depredadores mamíferos (M. S. Vazquez, en prensa). Todos los nidos estuvieron expuestos por un total de 12 días, que es el tiempo de incubación de la mayoría de las paseriformes (Mezquida and Marone 2001). Luego de ese período revisamos cada uno de los nidos y registramos el número de nidos depredados. Consideramos un nido depredado cuando al menos uno de los huevos no estuvo dentro del nido, o presentó signos de marcas o daños. A los huevos de plastilina los llevamos al laboratorio para evaluar las marcas de picos o dientes a fin de identificar a los depredadores.

\section{Análisis de datos}

Calculamos la tasa de depredación general de nidos como el número de nidos depredados dividido por el número total de nidos expuestos. Comparamos la depredación de nidos de acuerdo con la presencia de espinas usando un modelo lineal generalizado mixto con una distribución binomial. Por un lado, incluimos cada uno de los cuatro tratamientos como una variable explicativa. Por otro lado, planteamos un nuevo modelo incluyendo como variable explicativa la presencia/ausencia de espinas a dos niveles:

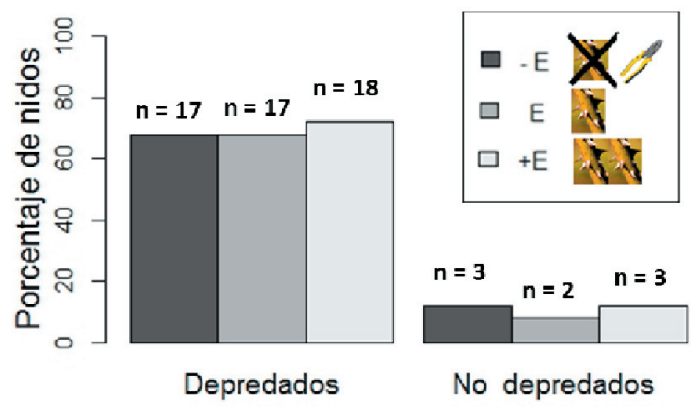

Figura 1. Porcentaje de nidos depredados y no depredados ubicados en arbustos de rosa mosqueta, en ramas con remoción manual de espinas (- E), espinosas (E) y con espinas agregadas $(+\mathrm{E})$.

Figure 1. Percentage of predated and non-predated nests located on rosa mosqueta shrubs, on branches free of thorns (-E), with thorns (E) and with addition of thorns $(+\mathrm{E})$.
1) micro-hábitat alrededor del nido (o sea, los tratamientos sobre la rosa mosqueta) y 2) en el vecindario (incluyendo el arbusto sin espinas). En todos los casos incluimos el sitio como factor aleatorio. Todos los análisis los desarrollamos usando el software R (R Core Team 2017).

\section{Resultados}

Del total de los 100 nidos expuestos, 18 no pudieron ser localizados al momento de la revisación, por lo cual se los eliminó del análisis. De los 82 restantes, 73 mostraron signos de haber sido depredados (i. e., $89 \%$ ). Los principales depredadores que dejaron marcas en los huevos de plastilina fueron ratones (16\%) y aves (4\%). Algunas de las marcas dejadas en la plastilina no pudieron ser identificadas (17\%) y en el resto de los casos el huevo de plastilina estuvo ausente o sin marcas (51\%). Finalmente, la probabilidad de depredación fue similar entre los cuatro tratamientos $\left(X^{2}=1.36, P=0.71\right)$, tanto analizando solo los tratamientos a nivel de micro-hábitat (i.e., en la rosa mosqueta), $\left(X^{2}=0.28, P=0.87\right)$ (Figura 1) como incluyendo al arbusto $\sin$ espinas $\left(X^{2}=1.15, P=0.28\right.$ ) (Figura 2).

\section{DisCUSIÓN}

Este trabajo presenta dos resultados principales: revela una alta tasa potencial de depredación y sugiere que las espinas no están protegiendo a los nidos de los depredadores. Primero, encontramos una tasa de depredación cercana a $90 \%$. Si bien este valor indica que

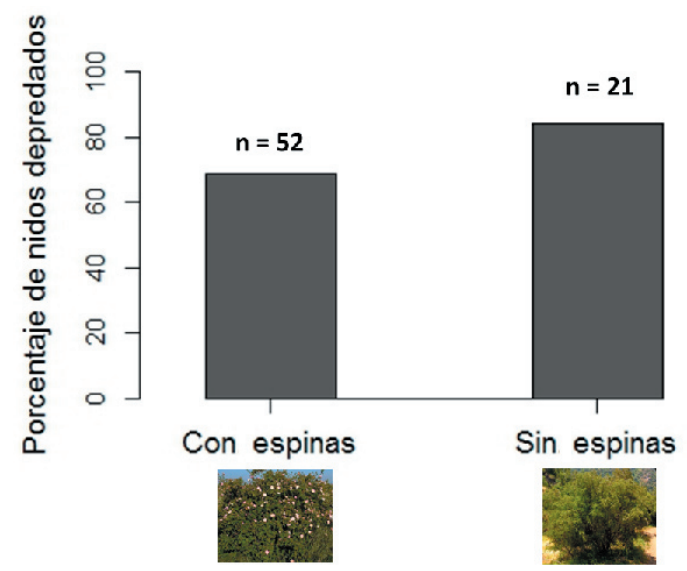

Figura 2. Porcentaje de nidos depredados ubicados en arbustos espinosos y arbustos completamente sin espinas.

Figure 2. Percentage of predated nests located in thorny shrubs and shrubs without thorns. 
la presión de depredación es una fuerza selectiva importante en la estepa patagónica, el mismo proviene de mediciones en nidos artificiales donde la ausencia de los padres puede influir sobre la tasa de depredación. Por ejemplo, la presencia de padres (esto es, sus olores, sonidos, movimientos, etc.) puede tanto ahuyentar como atraer depredadores (King et al. 1999; Burke et al. 2004; Faaborg 2004). Otro inconveniente de emplear nidos artificiales es que el olor humano y el material empleado para la construcción de los nidos pueden modificar el comportamiento de los depredadores (King et al. 1999). Sin embargo, en este estudio, los nidos artificiales fueron construidos y colocados con guantes y construidos con materiales que frecuentemente son los empleados por las especies de aves del área de estudio. En consecuencia, creemos que las tasas de depredación registradas son, al menos, un buen estimador de la importancia potencial de los depredadores de aves en estepas patagónicas. Esto es apoyado por el porcentaje de depredación que hallamos en nuestro trabajo, que coincide con los valores reportados tanto en estudios que emplean nidos artificiales como aquellos que miden depredación a campo en nidos naturales. Por ejemplo, nuestros valores son similares a los obtenidos con nidos artificiales en el monte argentino (Mezquida and Marone 2002) y en el bosque templado (Vazquez et al., en revisión). A su vez, estos resultados también coinciden con estudios en los que se estimaron las tasas de depredación en nidos naturales en diferentes regiones de Sudamérica (Lazo and Anabalón 1991; Mezquida and Marone 2001; Auer et al. 2007; Segura and Reboreda 2012). En cambio, el porcentaje de depredación detectado en nuestro trabajo es alto en comparación con los reportados para Norteamérica (Martin 1993). Esto puede reflejar las diferencias en las historias de vida de las aves, dado que las tasas de depredación son más altas para las especies tropicales y sudamericanas que para las norteamericanas (Martin 1996).

El segundo resultado relevante de nuestro estudio es que la presencia de espinas no afectó la depredación de nidos, ya que la probabilidad de que los huevos fuesen depredados fue independiente de la presencia y la abundancia de espinas, tanto alrededor del nido como en todo el arbusto. Esto sugiere que, al menos en el ambiente estudiado, ni las plantas espinosas ni la densidad de espinas alrededor de los nidos reducen la probabilidad de depredación. Por lo tanto, su ocurrencia/ abundancia no sería una característica que las aves seleccionen para reducir el riesgo de depredación de sus huevos. El papel de las espinas para reducir la depredación depende de la identidad de los depredadores. Como ya fuera discutido por otros autores, las espinas podrían limitar el acceso de algunos depredadores (Lazo and Anabalón 1991; Schmidt and Whelan 1999; Quader 2006) pero pueden favorecer el movimiento de otros. Por ejemplo, Borgmann and Rodewald (2004) mostraron que los mamíferos depredaron con mayor frecuencia nidos artificiales ubicados en plantas con espinas que en plantas sin espinas. En nuestro caso, la mayor cantidad de marcas en los huevos de plastilina fueron asignadas a roedores. Si bien el porcentaje hallado es menor a $20 \%$, es probable que el impacto de estas especies sea, en realidad, mayor y que aparezca subestimado en nuestro estudio debido al uso de plastilina, ya que estos pequeños mamíferos podrían olfatear los huevos de plastilina y, por lo tanto, evitar acercarse al nido. Además, la alta abundancia de roedores y la expansión de su distribución geográfica fue asociada a la introducción de arbustos exóticos (Pardiñas and Teta 2013). Es posible, entonces, que estos pequeños animales actúen como uno de los principales depredadores de huevos en la estepa patagónica y que las espinas no funcionen como protección contra estos depredadores.

Las características de micro hábitat alrededor del nido pueden afectar el riesgo de depredación (Martin 1995; Segura et al. 2012). Trabajos previos en ambientes similares a nuestro sitio de estudio revelaron resultados contrastantes en relación a la influencia de las espinas. En la región del Monte, nidos ubicados en especies no espinosas sufrieron tasas de depredación más bajas que aquellos ubicados en arbustos con espinas (Mezquida and Marone 2002). En el matorral chileno, la diuca (Diuca diuca) prefirió construir sus nidos sobre plantas sin espinas (Lazo and Anabalón 1991). Por otro lado, en ambientes similares, las aves usaron plantas espinosas para nidificar, a pesar de la mayor abundancia de plantas sin espinas; esto sugiere que la selección fue consecuencia de una presión de depredación más baja en las plantas con espinas (Mares et al. 1977). Los resultados de este trabajo también sugieren que la presencia de espinas no necesariamente 
reduce los niveles de depredación. Distintos factores influyen sobre la selección del sitio de nidificación, entre ellos la especie sustrato (Segura and Arturi 2009). La selección de determinadas especies vegetales podría estar más relacionada con las características estructurales de la planta que con la protección contra la depredación (Mezquida and Marone 2001). La selección de arbustos espinosos para nidificar podría entonces deberse a que la presencia de espinas facilitan la construcción de los nidos y le ofrecen una mayor estabilidad a los nidos terminados ante eventos climáticos como viento o lluvias fuertes que puedan ocasionar su caída (Marone et al. 1997). Futuras investigaciones deberían comparar el costo energético de construir nidos en arbustos con $\mathrm{y}$ sin espinas, $\mathrm{y}$ verificar experimentalmente si nidos construidos en ramas espinosas son realmente mas estables ante las inclemencias del tiempo.

Agradecimientos. A G. Pacheco, S. Quintas y L. Velazquez por su asistencia en el campo, a J. Pelliza por el abastecimiento de los huevos de canario y a G. Amico y L. Sympson por su revisión y aportes para mejorar el manuscrito. Los comentarios de dos revisores anónimos mejoraron la claridad de este manuscrito. S. Vazquez es becaria doctoral y AGFB investigador del CONICET. Este trabajo fue realizado dentro del marco de la materia Ecología de las Interacciones Planta-Animal dictada en Centro Regional Universitario Bariloche, Universidad Nacional del Comahue, Argentina.

\section{REFERENCIAS}

Aguiar, M. R., J. M. Paruelo, O. E. Sala, and W. K. Lauenroth. 1996. Ecosystem responses to changes in plant functional type composition: an example from the Patagonian steppe. Journal of Vegetation Science 7:381-390.

Auer, S. K., R. D. Bassar, J. J. Fontaine, and T. E. Martin. 2007. Breeding biology of passerines in a subtropical montane forest in northwestern Argentina. The Condor 109:321-333.

Biddle, L., A. M. Goodman, and D. C. Deeming. 2017. Construction patterns of birds' nests provide insight into nestbuilding behaviours. PeerJ 5:e3010.

Borgmann, K. L., and A. D. Rodewald. 2004. Nest predation in an urbanizing landscape: the role of exotic shrubs. Ecological Applications 14:1757-1765.

Burkart, R., N. O. Bárbaro, R. O. Sánchez, and D. A. Gómez. 1999. Eco-regiones de la Argentina. Presidencia de la Nación-Secretaría de Recursos Naturales y Desarrollo Sustentable-Administración de Parques Nacionales.

Burke, D. M., K. Elliott, L. Moore, W. Dunford, E. Nol, J. Phillips, S. Holmes, and K. Freemark. 2004. Patterns of nest predation on artificial and natural nests in forests. Conservation Biology 18:381-388.

Collias, N. E., and E. C. Collias. 2014. Nest building and bird behavior. Princeton University Press.

De la Peña, M. 2013. Nidos y reproducción de las aves argentinas. Serie Naturaleza, Conservación y Sociedad.

de La Vega, S. G., G. Carrizo, and A. Chaippe. 2003. Patagonia Las Leyes de La Estepa. Contacto Silvestre.

Faaborg, J. 2004. Truly artificial nest studies. Conservation Biology 18:369-370.

Filliater, T. S., R. Breitwisch, and P. M. Nealen. 1994. Predation on Northern Cardinal nests: Does choice of nest site matter? The Condor 96:761-768.

Janzen, D. H. 1969. Birds and the ant×acacia interaction in Central America, with notes on birds and other myrmecophytes. The Condor 71:240-256.

Joyce, F. J. 1993. Nesting success of rufous-naped wrens (Campylorhynchus rufinucha) is greater near wasp nests. Behavioral Ecology and Sociobiology 32:71-77.

Kelly, J. P. 1993. The effect of nest predation on habitat selection by Dusky Flycatchers in limber pine-juniper woodland. The Condor 95:83-93.

King, D. I., R. M. DeGraaf, C. R. Griffin, and T. J. Maier. 1999. Do Predation Rates on Artificial Nests Accurately Reflect Predation Rates on Natural Bird Nests?. Journal of Field Ornithology 70(2):257-262.

Lazo, I., and J. J. Anabalón. 1991. Nesting of the Common Diuca Finch in the central Chilean scrub. The Wilson Bulletin 103:143-146.

Lima, S. L. 2009. Predators and the breeding bird: behavioral and reproductive flexibility under the risk of predation. Biological Reviews 84:485-513.

Maier, T. J., and R. M. DeGraaf. 2000. Predation on Japanese Quail vs. House Sparrow eggs in artificial nests: small eggs reveal small predators. The Condor 102:325-332.

Mares, M., W. Blair, F. Enders, D. Greegor, A. Hulse, J. Hunt, D. Otte, R. Sage, and C. Tomoff. 1977. The strategies and community patterns of desert animals. Pp. 107-163 in G. H. Orians and O. T. Solbrig (eds.). Convergent evolution in warm deserts. Chapter 5. Dowden, Hutchison and Ross, Inc., Stroudsburg, Pennsylvania. Pp. 333.

Marone, L., J. Lopez de Casenave, and V. Cueto. 1997. Patterns of habitat selection by wintering and breeding granivorous birds in the central Monte desert, Argentina. Revista Chilena de Historia Natural 70:73-81. 
Martin, T. E. 1993. Nest predation among vegetation layers and habitat types: revising the dogmas. The American Naturalist 141:897-913.

Martin, T. E. 1995. Avian life history evolution in relation to nest sites, nest predation, and food. Ecological monographs 65:101-127.

Martin, T. E. 1996. Life history evolution in tropical and south temperate birds: what do we really know? Journal of Avian Biology 27:263-272.

Martin, T. E., and J. V. Briskie. 2009. Predation on dependent offspring. Annals of the New York Academy of Sciences 1168:201-217.

Matteucci, S. D. 2012. Ecorregión Estepa Patagónica. Pp. 549-654 in J. Morello, S. D. Matteucci, A. F. Rodríguez and M. Silva (eds.). Ecorregiones y complejos ecosistémicos argentinos. Orientación Gráfica Editora SRL, Buenos Aires.

Mezquida, E. T., and L. Marone. 2001. Factors Affecting Nesting Success of a Bird Assembly in the Central Monte Desert, Argentina. Journal of Avian Biology 32:287-296.

Mezquida, E. T., and L. Marone. 2002. Microhabitat structure and avian nest predation risk in an open Argentinean woodland: an experimental study. Acta Oecologica 23:313-320.

Pardiñas, U. F., and P. Teta. 2013. Holocene stability and recent dramatic changes in micromammalian communities of northwestern Patagonia. Quaternary International 305:127-140.

Povedano, H. E., and M. V. Bisheimer. 2016. Aves terrestres de la Patagonia: Tierra del Fuego e Islas del Atlántico Sur. María Victoria Bisheimer.

Quader, S. 2006. What makes a good nest? Benefits of nest choice to female Baya Weavers (Ploceus philippinus). The Auk 123:475-486.

R Core Team. 2017. R: A Language and Environment for Statistical Computing. R Foundation for Statistical Computing, Vienna, Austria.

Schmidt, K. A., and C. J. Whelan. 1999. Effects of exotic Lonicera and Rhamnus on songbird nest predation. Conservation Biology 13:1502-1506.

Segura, L. N., and M. F. Arturi. 2009. Selección de sitios de nidificación del Cardenal Común (Paroaria coronata) en bosques naturales de Argentina. Ornitología Neotropical 20:203-213.

Segura, L. N., D. A. Masson, and M. G. Gantchoff. 2012. Microhabitat nest cover effect on nest survival of the Redcrested Cardinal. The Wilson Journal of Ornithology 124:506-512.

Segura, L. N., and J. C. Reboreda. 2012. Nest survival rates of Redlcrested Cardinals increase with nest age in south temperate forests of Argentina. Journal of Field Ornithology 83:343-350.

Smith, H. M., C. R. Dickman, and P. B. Banks. 2016. Nest predation by commensal rodents in urban bushland remnants. PloS one 11:e0156180.

Young, B. E., M. Kaspari, and T. E. Martin. 1990. Species-specific nest selection by birds in ant-acacia trees. Biotropica 22:310-315. 